\title{
A New Model for the Evolution of Carnivory in the Bladderwort Plant (Utricularia): Adaptive Changes in Cytochrome c Oxidase (COX) Provide Respiratory Power
}

\author{
L. Laakkonen ${ }^{1}$, R. W. Jobson ${ }^{2}$, and V. A. Albert ${ }^{3}$ \\ ${ }^{1}$ Helsinki Bioenergetics Group, Programme for Structural Biology and Biophysics, Institute of Biotechnology, Biocenter 3 (Viikinkaari 1), \\ PB 65, University of Helsinki, 00014 Helsinki, Finland \\ ${ }^{2}$ Department of Ecology and Evolutionary Biology, 2052 Kraus Natural Science Bldg., 830 N. University, Ann Arbor, MI 48109-1048, USA \\ ${ }^{3}$ Natural History Museum, University of Oslo, P.O. Box 1172 Blindern, 0318 Oslo, Norway
}

Received: December 28, 2005; Accepted: June 30, 2006

\begin{abstract}
The evolution of carnivorous plants has been modeled as a selective tradeoff between photosynthetic costs and benefits in nutrient-poor habitats. Although possibly applicable for pitfall and flypaper trappers, more variables may be required for active trapping systems. Bladderwort (Utricularia) suction traps react to prey stimuli with an extremely rapid release of elastic instability. Trap setting requires considerable energy to engage an active ion transport process whereby water is pumped out through the thin bladder walls to create negative internal pressure. Accordingly, empirical estimates have shown that respiratory rates in bladders are far greater than in leafy structures. Cytochrome $c$ oxidase (COX) is a multi-subunit enzyme that catalyzes the respiratory reduction of oxygen to water and couples this reaction to translocation of protons, generating a transmembrane electrochemical gradient that is used for the synthesis of adenosine triphosphate (ATP). We have previously demonstrated that two contiguous cysteine residues in helix 3 of COX subunit I (COX I) have evolved under positive Darwinian selection. This motif, absent in $\approx 99.9 \%$ of databased COX I proteins from eukaryotes, Archaea, and Bacteria, lies directly at the docking point of COX I helix 3 and cytochrome $c$. Modeling of bovine COX I suggests the possibility that a vicinal disulfide bridge at this position could cause premature helix termination. The helix 3-4 loop makes crucial contacts with the active site of COX, and we postulate that the $\mathrm{C}-\mathrm{C}$ motif might cause a conformational change that decouples (or partly decouples) electron transport from proton pumping. Such decoupling would permit bladderworts to optimize power output (which equals energy times rate) during times of need, albeit with a $20 \%$ reduction in overall energy efficiency of the respiratory chain. A new model for the evolution of bladderwort carnivory is proposed that includes respiration as an additional tradeoff parameter.
\end{abstract}

Key words: Bladderworts, carnivorous plants, cytochrome c oxidase, conformational change, COX, electron transport, energetics, protein structure, proton pumping, respiration, Utricularia.

Plant Biol. 8 (2006): $758-764$

(C) Georg Thieme Verlag KG Stuttgart · New York

DOI 10.1055/s-2006-924459

ISSN $1435-8603$

\author{
Abbreviations: \\ COX: \\ COX I: \\ cOX II: \\ cyt c: \\ ATP synthase: \\ cytochrome c oxidase \\ cytochrome $c$ oxidase subunit I \\ cytochrome $c$ oxidase subunit II \\ cytochrome $c$ \\ adenosine triphosphate synthase
}

\section{Introduction}

Carnivorous plants exist in a delicate balance between the costs and benefits of nutrient acquisition (Givnish, 1989; Juniper, 1989; Ellison and Gotelli, 2001). Trapping structures that are often less photosynthetic may stress carnivores in terms of carbon production (Juniper, 1989; Adamec, 1997). However, nutrients acquired from prey can offset photosynthetic limitations imposed by carnivory (Givnish, 1989; Juniper 1989; Méndez and Karlsson 1999; Ellison and Gotelli, 2001; Wakefield et al., 2005). Givnish (1984) modelled carnivorous plant evolution in terms of photosynthetic costs/benefits in nutrient-poor habitats. According to this model, nutrient acquisition by carnivory can be adaptive via higher photosynthetic rates in moist, sunny environments. The Givnish framework has been a useful one (Ellison, 2001), but it does not generalize well to all carnivorous trapping systems. Plants that invest resources in passive traps, such as pitfall and flypaper devices (e.g., Sarracenia and Drosera, respectively), may indeed have photosynthesis as their greatest limitation, but active trappers also invest energy (Juniper, 1989; Knight, 1992; Adamec, 2006). The extent to which energy investment is required depends upon the degree of activity involved.

In the venus flytrap, Dionaea, fluid movement is required for establishment of leaf curvature, the geometry of which is solely responsible for trap closure after triggering (Hodick and Sievers, 1989; Forterre et al., 2005). In bladderworts, Utricularia, active water transport generates negative pressure within the thin-walled suction traps, but triggering involves a release of elastic instability upon pressure equilibration (Sydenham and Findlay, 1973 a; Juniper et al., 1989; Skotheim and Mahadevan, 2005). Whereas the biochemical-hydrodynamic mechanism through which Dionaea leaves attain curvature is only poorly known, bladderworts transport water out of their traps via a two-step ATP-driven ion pumping process, initiated by 
respiration-dependent transport of chloride ions against an electrochemical gradient (Sydenham and Findlay, 1973a,b, 1975; Fineran and Lee, 1980; Juniper et al., 1989). This active water removal process likely requires considerably more energy than the relatively gradual reopening of Dionaea leaves that follows prey digestion (Sydenham and Findlay, 1975; Juniper et al., 1989). Physiological studies (Adamec, 2006) show that respiration in Utricularia bladders is $75-200 \%$ greater than in leaf-like structures, whereas photosynthetic rates are $7-$ 10-times lower. As such, maintenance of bladder traps constitutes a significant metabolic tradeoff between energy and carbon production (Adamec, 2006).

Previously, we have reported explicit molecular evolutionary evidence that the rate-limiting enzyme in the cellular respiration pathway, cytochrome $c$ oxidase (COX) (Saraste, 1999), may be functionally altered in bladderworts (Jobson et al., 2004). We identified 12 amino acid sites in subunit I of COX (COX I) that showed evidence for positive Darwinian selection in the lineage that includes Utricularia and its sister group, the corkscrew plant, Genlisea. These changes were absent in the butterworts, Pinguicula, which we and others have found to be the sister clade to Utricularia plus Genlisea (Jobson and Albert, 2002; Jobson et al., 2003; Müller et al., 2004). Two of the positively selected sites are contiguous and lie near the end of COX I helix 3. In bladderworts (and one Genlisea species), a double cysteine $(\mathrm{C}-\mathrm{C})$ motif substituted for small, hydrophobic residues otherwise known from many organisms. No other cysteines were found in the vicinity. We hypothesized that the two cysteines could form a vicinal disulfide bridge under some conditions, probably causing helix 3 to unravel prematurely. Although vicinal disulfides have not been reported in alpha helices, we successfully modelled the possibility in silico based on the bovine COX enzyme. Our proposal was that premature termination of COX I helix 3 could lead to changes in the proposed docking base (Roberts et al., 1999; Flöck and Helms, 2002) for the electron carrier cytochrome $c$ (cyt c), which must associate/disassociate with the COX holoenzyme four times to reduce an $\mathrm{O}_{2}$ molecule, as COX generates a potential difference worth 8 unit charges over the inner mitochondrial membrane. The electrochemical potential generated is used to power ATP synthesis. Specifically, we hypothesized that docking base changes between COX and cyt $c$ could alter their association/dissociation kinetics to affect the rate of proton pumping and therefore the rate of ATP production. We supposed that unravelling of COX I helix 3 might modify the surface area of the COX/cyt $c$ docking base, thereby increasing the kinetics of respiration (unpublished).

Here, we propose a second mechanism whereby the C-C motif might affect bladderwort energetics. The hypothetical Utricularia disulfide could disturb the conserved loop between COX I helices 3 and 4, which is in contact with the active site of the holoenzyme. We consider the possible effects of changed interactions at the active site, and their implications for the evolution of bladderwort carnivory in terms of energetic as well as photosynthetic tradeoffs. The two hypotheses we present are not mutually exclusive, and could well act in concert. They are discussed separately because they refer to different mechanisms.

\section{Materials and Methods}

CHARMM C30B1 (Brooks et al., 1983) was used for protein structural modelling. Protein models were visualized by using VMD 1.8.2 (Humphrey et al., 1996).

\section{Results and Discussion}

\section{Cytochrome c oxidase (COX): a review}

The vast majority of living organisms depend on the ability to use $\mathrm{O}_{2}$ as the acceptor of electrons harvested during the citric acid cycle. The terminal respiratory enzymes in aerobic prokaryotes and all eukaryotes are called heme-copper oxidases (EC 1.9.3.1), after their active site composed of two hemes and a copper atom. They are large membrane protein complexes, whose three central subunits are homologous in all kingdoms of life (Saraste, 1999). In eukaryotes, the central subunits are encoded by the mitochondrial genome, whereas the additional 4-10 subunits are nuclear-encoded, and their function is largely unknown (Fig.1) (Kadenbach, 2003). The only absolutely conserved amino acids in the enzyme are the ligands of the metal ions, which indicates that the electron transfer paths are identical, as is fitting for extra-fast tunnelling effects. However, electron entry to the catalytic subunit does vary, as do the structures for proton channels and clearly also the mobile parts of the active site that expel the reaction product, water.

The catalytic reaction, $\mathrm{O}_{2}+8 \mathrm{H}^{+}$in $+4 \mathrm{e}^{-} \rightarrow 2 \mathrm{H}_{2} \mathrm{O}+4 \mathrm{H}^{+}$out, takes place inside the membrane-embedded parts of the protein, and produces a charge and $\mathrm{pH}$ difference, the proton motive force $\left(\Delta \mu_{\mathrm{H}^{+}}\right)$, across the membrane; first, because the charged reagents are taken up from opposite sides of the membrane (Mitchell, 1961, 1966) and, second, because additional protons are pumped across the membrane (Wikström, 1977). The built-up potential opposes further reaction, and then the activity of the respiratory chain slows down. In the cell/mitochondria, $\Delta \mu_{\mathrm{H}^{+}}$drives several transport processes, and most importantly, it powers ATP synthase. For maximal power output of ATP production, partial decoupling of catalysis from pumping is beneficial, as this eliminates the back pressure caused by $\Delta \mu_{\mathrm{H}^{+}}($Stucki, 1980).

Electrons are donated to COX by cytochrome $c$ (cyt $c$ ), a small protein on the +-side of the membrane. Cyt $c$ is guided to its docking site by electrostatic forces, and forms a transient complex with the oxidase for electron transfer. The slowest and rate-determining step in the electron transfer process is the dissociation of this donor-acceptor complex (Ferguson-Miller et al., 1976). Protons enter the active site via hydrogen-bonded water chains found in protein cavities. The channels are experimentally well defined and are called D and K channels, for their invariable amino acids (Saraste, 1999). Crystal structures of bovine heart COX and four bacterial oxidases are known (Ostermeier et al., 1997; Abramson et al., 2000; Soulimane et al., 2000; Svensson-Ek et al., 2002; Tsukihara et al., 2003). The number of nuclear-encoded subunits (Fig. 1) varies from animals to plants to fungi, and the sequences are highly divergent. Their role is not clear, but the fact that several of them have tissue- and developmental stage-specific isoforms indicates a regulatory role in catabolism (Burke and Poyton, 1998). 


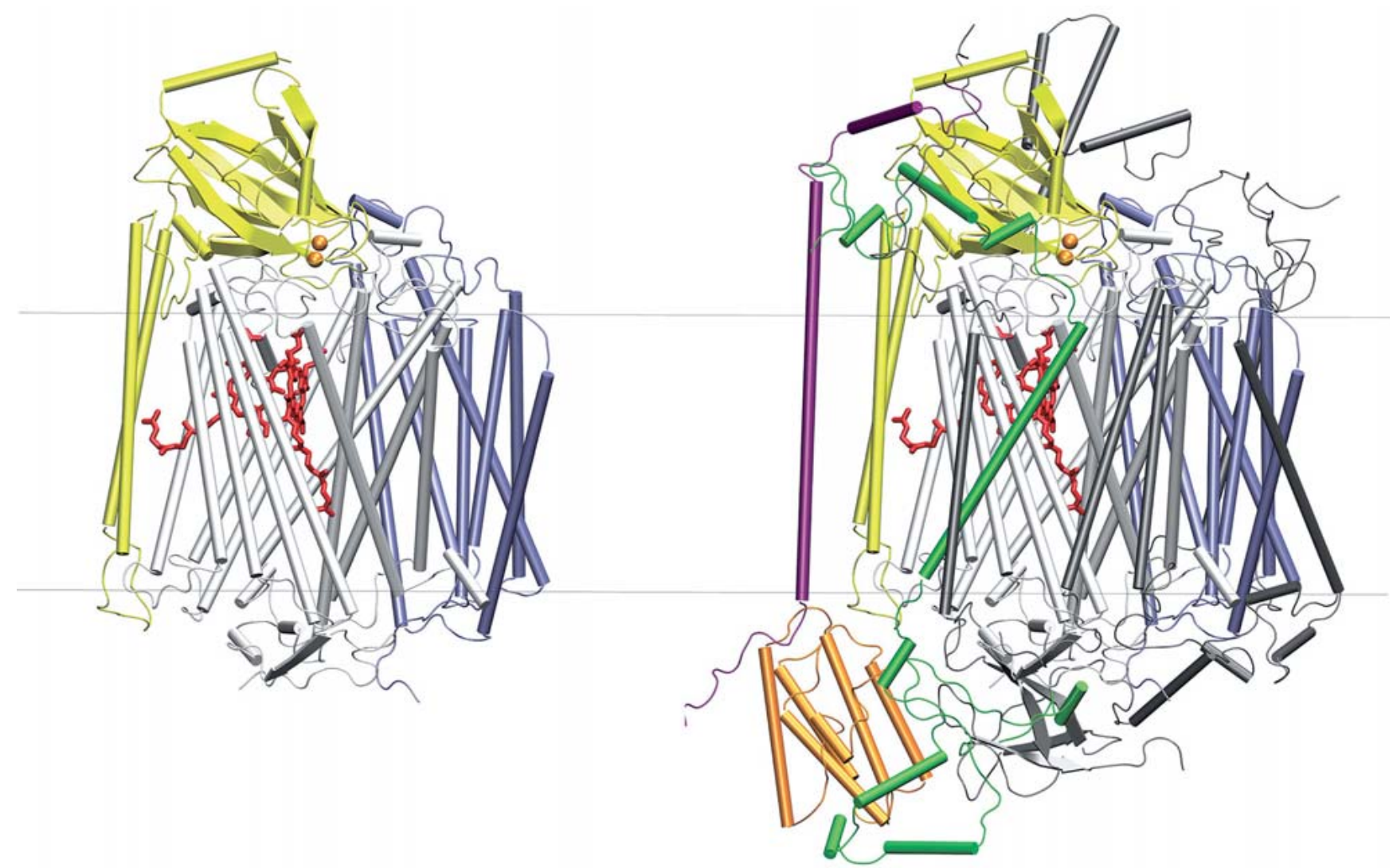

Fig. 1 Bovine cytochrome c oxidase. Left, core subunits only, right, all 10 subunits. COX I is shown in silver, heme groups in red, and copper ions as orange dots. COX II is coloured yellow, and COX III pale blue. Three nuclear subunits are highlighted: COX IV (green), COX Va

Regulation of COX could occur by two conceptually different ways that are not mutually exclusive. First, if it were possible to change the dissociation kinetics of cyt $c$, the reaction rate could be easily enhanced or retarded. Structurally, this translates to changes in the docking base of cyt $c$. No docked complex has been isolated experimentally, but there are computed structures that agree with mutational and spectroscopic data (Roberts et al., 1999; Flöck and Helms, 2002). Cyt $c$ is in contact with several of the nuclear subunits, while the largest contact areas are to COX I and COX II. Another possibility to regulate would be to loosen the coupling between catalysis and pumping and, in this way, increase the rate of ATP production at the cost of efficient use of reductive equivalents (Stucki, 1980).

\section{The C-C motif in COX I: structural and taxonomic disposition}

Among the 12 amino acid sites we determined to be under positive Darwinian selection in bladderworts is a double cysteine (C-C) motif near the C-terminus of COX I helix 3. This motif corresponds to Leu-113-Ser-114 in the bovine enzyme. As we reported previously, no other cysteine residues lie nearby in the structure, including in the nuclear-encoded subunit COX VIIa (Fig. 2; unpublished data). COX VIIc, which together with nuclear-encoded COX VIIa and mitochondrially-encoded COX I forms a "tripod"-like interaction point near the proposed docking base of cyt c (Fig. 2), is not known in plants. (orange), and COX VIc (purple); the rest are coloured grey. The actual membrane is not visible in the crystal structures, but approximate membrane level is marked in the figure, intermembrane space up, mitochondrial matrix down.
The C-C motif is common to all investigated bladderwort species, one corkscrew plant taxon, and the phylogenetically isolated gymnosperm, Welwitschia. No other carnivorous plant families have the motif, and neither does it appear in any other of the $>33000$ COX I sequences currently databased for eukaryotes, Archaea, and Bacteria. Of the investigated corkscrew plant species, Genlisea hispidula has C-C, Genlisea aurea has C-S, whereas Genlisea violacea has L-S, like Pinguicula and asterids. Genlisea, the sister genus to Utricularia, may have differentially lost the $\mathrm{C}-\mathrm{C}$ motif after loss of selection pressure for active water transport (Jobson et al., 2004). This model assumes an active trapping ancestor for both genera.

\section{Hypothesis 1: Bladderwort COX is regulated by altered cytochrome c association-dissociation kinetics}

We have previously hypothesized that a vicinal disulfide bridge at the bladderwort C-C motif could lead to early termination of helix 3 , thereby altering the tripod-like region and cyt $c$ docking base. A reversible (or non-reversible) conformational change in the docking base could theoretically lower the surface area for cyt $c$ association-dissociation and upregulate COX/cyt $c$ kinetics. A similar functional hypothesis has been suggested by Schmidt et al. (2005) for COX/cyt $c$ in anthropoid primates. A number of amino acid residues in both cyt $c$ and COX appear to have co-evolved with charge-neutralizing amino acid replacements at the docking base. Reduced electro- 


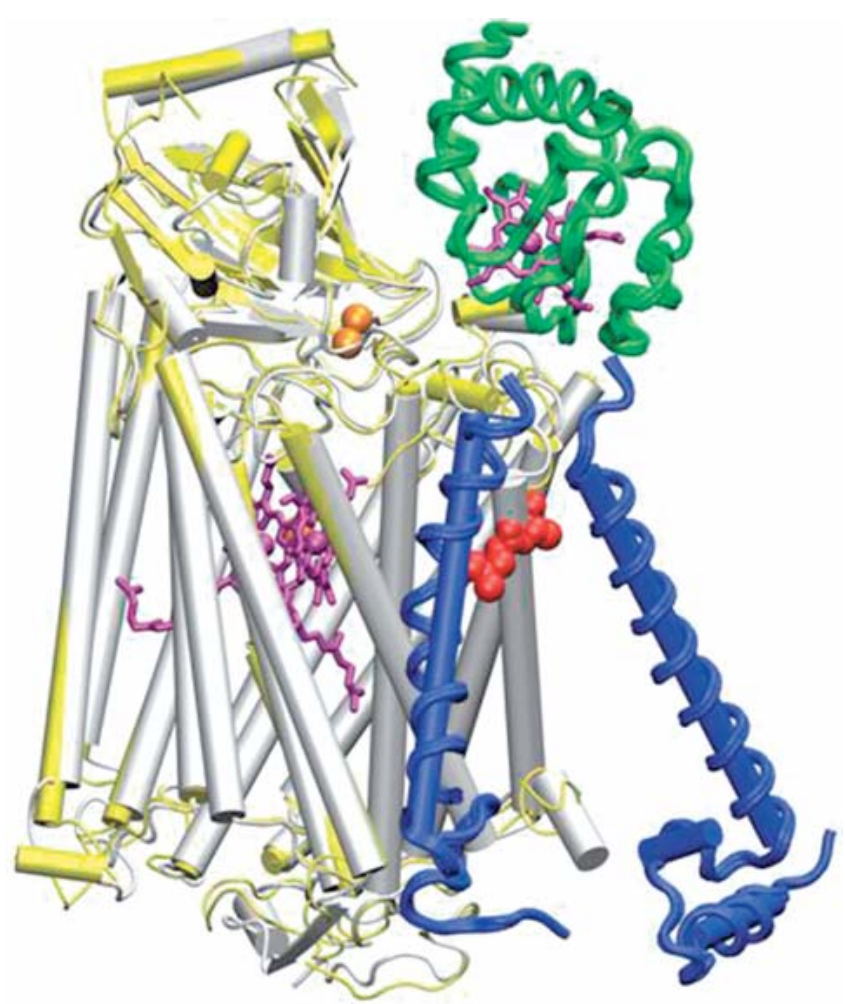

Fig. 2 Protein structural reconstruction of COX I and the nuclear subunits COX VIla and VIIc based on the bovine enzyme model. COX I is in silver, and hemes and coppers show through in purple and orange, respectively. COX subunits VIIa, VIIc are shown in dark blue. COX VIIa exists in plants, including Utricularia. Leu-113-Ser-114 of bovine I are shown as red space-filling models. Cytochrome $c$, in green, is shown superimposed by using coordinates from the $P$. denitrificans oxidase (1ar1.pdb)/cytochrome $c$ docked complex. COX I residues 113 and 114 and the C termini of COX VIIa, VIIc form a "tripod" directly at the cytochrome $c$ docking base. See Jobson et al. (2004).

static interaction between the proteins could then upregulate dissociation kinetics. The basic concurrence between these hypotheses is attractive, yet both lack the necessary experimental proof; for example, direct measurements of reaction rates in the targeted organisms, or measurements of COX kinetics in mutagenized yeast.

Hypothesis 2: Bladderwort COX is regulated by decoupling of electron transport and proton pumping

The C-C motif in COX I helix 3 lies very near the loop between helices 3 and 4 (Fig. 2), which is extremely well conserved among eukaryotes. In the bovine structure, the loop dips deep into the helix bundle, and the innermost tryptophan (Trp) forms a hydrogen bond to heme $a_{3}$ (Figs. 3,4 ). There is also a conserved double proline motif in cis conformation in the loop, in contact with COX subunit II (COX II; Fig. 4). Intriguingly, mutation of the Trp residue is known to affect proton pumping (Ribacka et al., 2005). The same loop 3-4 is dramatically different between mitochondrial oxidases and their distant bacterial relatives, the $c b b_{3}$ oxidases, strongly suggesting an important functional role (Sharma et al., 2006).

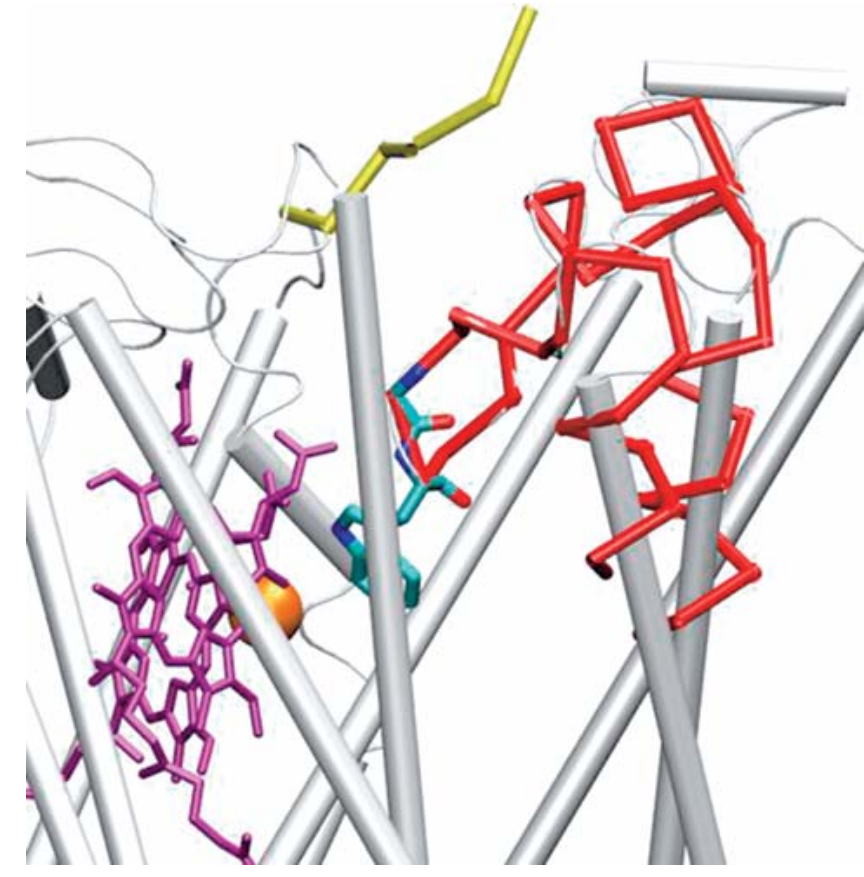

Fig. 3 Loop 3-4 of COX I extends deep into the COX active site. The carbon backbone of the loop and helix ends is shown in red. The terminal Trp residue, in aqua, makes contact with heme $a_{3}$. The yellow carbon chain is part of nearby COX II. Other structural elements are as in Fig. 1.

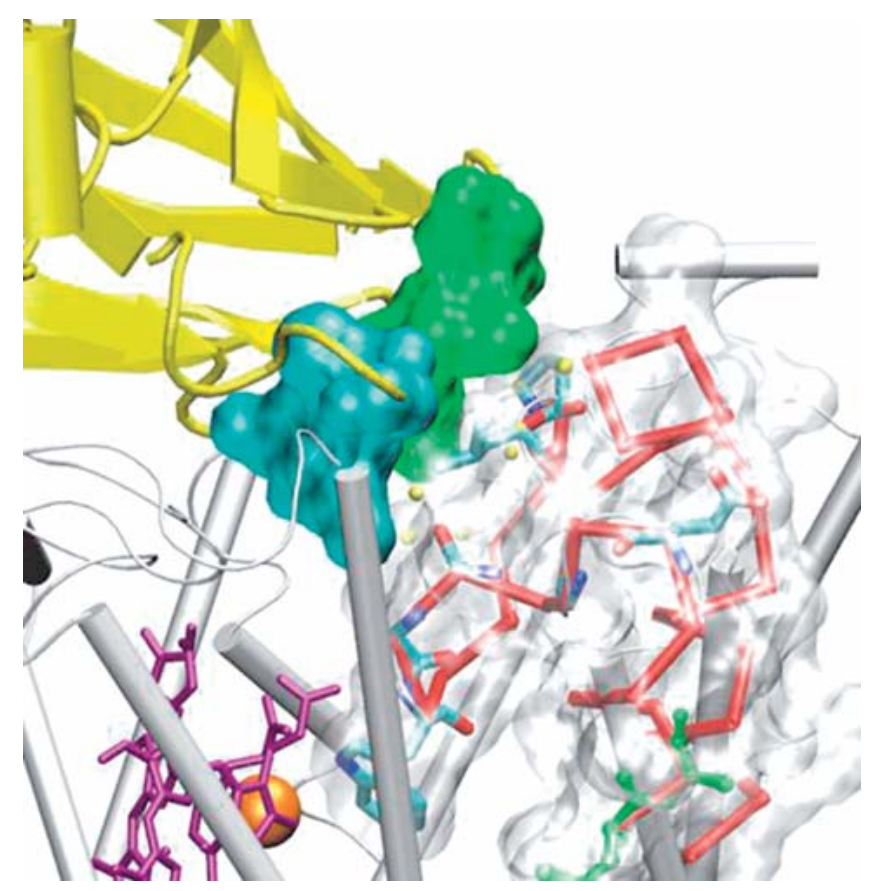

Fig. 4 Loop 3-4, shown as a transparent space-filling model. The carbon backbone shows through in red, and the C-C motif on helix 3 is in green. COX II is shown in cartoon (yellow) except for two regions (aqua and green, respectively) that independently contact loop 3-4 via a double proline motif. 
It is perhaps significant that in silico modelling suggests only $1-2$ amino acids would be required to construct a loop 3-4 that had no other function (unpublished). The potential structural implication is that free cysteines would form a longer helix, whereas a disulfide would terminate the helix about two turns earlier (as above), causing a conformational change at $\operatorname{Trp} /$ heme $a_{3}$ that leads to uncoupling of proton pumping from electron transport. Moreover, this hypothetical conformational change at loop 3-4 would still be expected to alter the COX/cyt $c$ docking base, as in Hypothesis 1.

\section{Functional implications}

Implicit in Hypothesis 1 is that electron transport and proton pumping are still coupled despite increased COX/cyt $c$ association-dissociation rate. As such, overall energy production could be increased, but only by a constant linked to this protein interaction rate. Molecular evolutionary changes permitting increased COX/cyt $c$ association-dissociation rates would be fixed for the lifetime of the organism, as opposed to permitting physiologically-induced flexibility.

Hypothesis 2, which is our preferred hypothesis based on structural evidence, could permit fine scale, physiologicallybased regulation of energetic power, albeit at an overall energy cost, via the possibility for total or partial uncoupling of electron transport from proton pumping. If complete decoupling occurred and the enzyme no longer pumped protons, one charge would still be transferred across the membrane for the electron and proton needed in water formation. At five charges total per the respiratory chain, the net loss is $20 \%$. Despite the overall loss in energetic efficiency, decoupling could permit $\mathrm{H}^{+}$ ions to build up a positive energy potential in the intermembrane space that is independent from the electron transport rate. Since ATP synthase interconverts the proton motive force, $\Delta \mu_{\mathrm{H}^{+}}$to ATP, greater $\Delta \mu_{\mathrm{H}^{+}}$would mean greater ATP production. Via a conformational change at loop 3-4, an organism could then fine-tune the speed of ATP production to needs, albeit at some cost to overall efficiency. With experimental evidence showing respiration in active trapping Utricularia bladders to be $75-200 \%$ greater than in the passive leaf-like structures (Adamec, 2006), Hypothesis 2 suggests that decoupling is spatially regulated in bladderworts, perhaps by expressing different nuclear-encoded isoforms in different tissues. In mammals, differential expression of such isoforms is known to have regulatory implications (e.g., Grossman et al., 2001). While nothing is known of COX isoforms in plants, in the bovine enzyme, loop 3-4 is implicated in the tissue-specific isoform differences of the small subunits in mammals (unpublished).

\section{Adaptive tradeoffs and a model for the evolution of bladderwort carnivory}

The massive respiratory output of Utricularia traps places a severe metabolic constraint on the plant, which can have bladders as a significant portion of its biomass (Juniper et al., 1989; Friday, 1992; Guisande et al., 2000, 2004; Richards, 2001). The metabolic cost of traps is made all the more severe by their 7-10-times lower photosynthetic capacity relative to leaf-like structures (Adamec, 2006). Clearly, bladderworts "walk a fine line" in terms of adaptive tradeoffs.

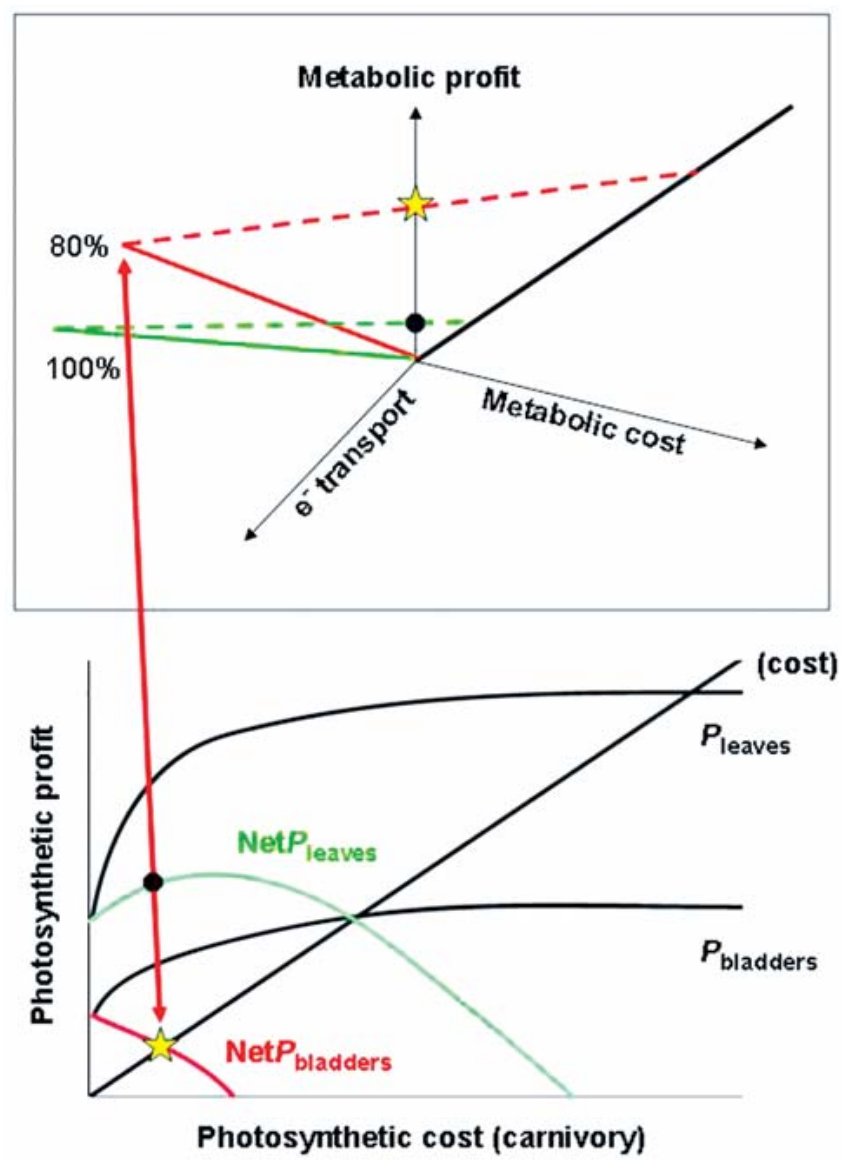

Fig. 5 Model for the evolution of bladderwort carnivory including both photosynthesis and respiration as parameters. See text for details.

Givnish's (1984) model for carnivorous plant evolution was parameterized in terms of photosynthetic cost/profit in nutrient-poor habitats. Investment of carbon in elaborate traps must be balanced by nutrient gain. Passive- or even moderately active-trapping carnivores may indeed have photosynthetic capacity as their principal limitation, but the highly activetrapping bladderwort requires - and has been demonstrated to use - massive amounts of energy (Sydenham and Findlay, 1973 a; Adamec, 2006).

We present a new model for the evolution of bladderwort carnivory in Fig. 5. The lower graph follows the Givnish model and its emphasis on photosynthetic rates (see Ellison, 2001). Altered from that model are the labels on the curves representing photosynthetic rates. Givnish $(1984,1989)$ considered rates in moist, high-light habitats (upper black curve) versus those in dryer, low-light environments (lower black curve). The green and red curves, respectively, represented net photosynthetic profits (rates minus costs [diagonal]) given the photosynthetic costs of carnivory. Givnish envisioned carnivory to become adaptive in terms of cost-benefit tradeoffs when the net profit curve for moist, high-light habitats sloped upward (roughly speaking, at the black dot on the green curve). For our purposes with Utricularia, and based on empirical data (Adamec, 2006), we merely replace photosynthetic rate differences between moist, high-light versus dryer, low-light habitats with those for leaves versus bladders (respectively). Im- 
mediately apparent is that the photosynthetic cost incurred by producing bladders does not favour their evolution under Givnish's $(1984,1989)$ model (yellow star). However, as we have argued, bladderwort respiration must also become an evolutionary parameter.

The boxed graph at the top of Fig. 5 represents bladderwort respiration via three axes, two of which correspond to the photosynthetic graph below. In parallel with photosynthesis, metabolic cost and metabolic profit are evolutionary tradeoffs for bladderworts. The third axis on the graph is overall energy produced by the respiratory chain, expressed in terms of efficiency. Under Hypothesis 2, reduced overall energy efficiency (metabolic cost), with or without uncoupling of electron transport from proton pumping (i.e., $80 \%$ vs. $100 \%$ efficiency), must be balanced with the gain in nutrients conferred by carnivory (metabolic gain). The solid green and red lines track total energy along the $\mathrm{e}^{-}$axis, and the like-coloured dashed lines intercept the metabolic cost/benefit diagonal as follows: (1) $100 \%$ (green) incurs low metabolic cost, but the metabolic investment is insufficient to make bladderwort carnivory adaptive (black dot). (2) $80 \%$ (red) incurs a higher metabolic cost, but the gain in terms of respiratory power outweighs it (yellow star).

Given that the common ancestor of the carnivorous butterworts (Pinguicula), corkscrew plants (Genlisea) and bladderworts (Utricularia) was very likely to have been a flypaper trapper (Albert et al., 1992), overcoming the net photosynthetic cost of carnivory (black dot in lower graph) would have predated evolution of the bladder mechanism. As such, we hypothesize that metabolic tradeoffs would have become the most important parameters during the evolution of bladders. The red line between the graphs illustrates this relative parameter importance.

\section{Conclusions}

If our structural and mechanistic hypotheses are correct, the nearly unique $\mathrm{C}-\mathrm{C}$ motif in bladderwort COX I may have significant functional and evolutionary implications:

1. Early termination of COX I helix 3 by a vicinal disulfide bridge could alter the functionally vital loop 3-4, which makes direct contact with the COX active site. Such a conformational change could alter the COX/cyt $c$ docking base or inactivate proton pumping, the latter decoupling proton pumping from electron transport.

2. Fine-tuned regulation of proton motive force, $\Delta \mu_{\mathrm{H}^{+}}$, would, in turn, permit fine-tuned regulation of ATP production, i.e. at times and in tissues where it is needed the most.

3. Respiratory regulation in this manner could be responsible for greater respiration in the bladders of Utricularia versus their leaf-like structures.

4. Given that photosynthesis in bladders in much less than in leaf-like structures despite the bladders' significant contribution to biomass, metabolic versus photosynthetic gain must exist in a very delicate balance.

5. A new model for bladderwort carnivory that assumes carnivory itself evolved first in the common ancestor of the butterworts, corkscrew plants, and bladderworts, suggests that metabolic gain permitted by optimization of energetic power was the decisive scaffolding parameter underlying bladder evolution from non-active trapping structures.
Future research would first require isolation and characterization of Utricularia COX. We would need the protein complex both from leaves and bladders to be able to assign the observed tissue-level difference in respiratory activity (Adamec, 2006) unequivocally to the function of cytochrome $c$ oxidase. Further, the existence of the $\mathrm{C}-\mathrm{C}$ vicinal disulfide should be addressed with mass spectrometry. Another useful experiment could be in vitro mutagenesis to produce the $\mathrm{C}-\mathrm{C}$ motif in yeast COX I, followed by physical and physiological characterization. Further research on nuclear COX subunits in plants could also be illuminating.

\section{Acknowledgements}

We thank Mårten Wikström for many fruitful discussions of ideas, and an anonymous reviewer for suggestions to improve the manuscript. L. L. thanks the Academy of Finland (Center of Excellence grant 1202898) for funding.

\section{References}

Abramson, J., Riistama, S., Larsson, G., Jasaitis, A., Svensson-Ek, M., Laakkonen, L., Puustinen, A., Iwata, S., and Wikstrom, M. (2000) The structure of the ubiquinol oxidase from Escherichia coli and its ubiquinone binding site. Nature Structural Biology 7, 910-917.

Adamec, L. (1997) Photosynthetic characteristics of the aquatic carnivorous plant Aldrovanda vesiculosa. Aquatic Botany 59, $297-$ 306.

Adamec, L. (2006) Respiration and photosynthesis of bladders and leaves of aquatic Utricularia species. Plant Biology 8, 765-769.

Albert, V. A., Williams, S. E., and Chase, M. W. (1992) Carnivorous plants: phylogeny and structural evolution. Science 257, 1491 1495.

Brooks, B. R., Bruccoleri, R. E., Olafson, B. D., States, B. J., Swaminathan, S., and Kaplus, M. (1983) CHARMM: a program for macromolecular energy, minimization, and dynamics calculations. Journal of Computational Chemistry 4, 187-217.

Burke, P. V. and Poyton, R. O. (1998) Structure/function of oxygenregulated isoforms in cytochrome $c$ oxidase. Journal of Experimental Biology 201, $1163-1175$.

Ellison, A. M. and Gotelli, N. J. (2001) Evolutionary ecology of carnivorous plants. Trends in Ecology and Evolution 16, 623 - 629.

Ferguson-Miller, S., Brautigan, D. L., and Margoliash, E. (1976) Correlation of the kinetics of electron transfer activity of various eukaryotic cytochromes $c$ with binding to mitochondrial cytochrome $c$ oxidase. Journal of Biological Chemistry 251, 1104-1115.

Fineran, B. A. and Lee, M. S. L. (1980) Organization of mature glands on the trap and other organs of the bladderwort Utricularia monanthos. Protoplasma 103,17-34.

Flöck D. and Helms, V. (2002) Protein-protein docking of electron transfer complexes: cytochrome $c$ oxidase and cytochrome $c$. Proteins $47,75-85$.

Forterre, Y., Skotheim, J. M., Dumais, J., and Mahadevan, L. (2005) How the Venus flytrap snaps. Nature 433, 421 - 425.

Friday, L. E. (1992) Measuring investment in carnivory: seasonal and individual variation in trap number and biomass in Utricularia vulgaris L. New Phytologist 121, $439-445$.

Givnish, T. J. (1989) Ecology and evolution of carnivorous plants. In Plant-Animal Interactions (Abrahamson, W. G., ed.), New York: McGraw-Hill, pp. $242-290$.

Givnish, T. J., Burkhardt, E. L., Happel, R. E., and Weintraub, J. D. (1984) Carnivory in the bromeliad Brocchinia reducta, with a cost/ benefit model for the general restriction of carnivorous plants to sunny, moist nutrient-poor habitats. American Naturalist 124, $479-497$. 
Grossman, L. I., Schmidt, T. R., Wildman, D. E., and Goodman, M. (2001) Molecular evolution of aerobic energy metabolism in primates. Molecular Phylogenetics and Evolution 18, 26-36.

Guisande, C., Andrade, C., Granado-Lorencio, C., Duque, S. R., and Nunez-Avellaneda, M. (2000) Effects of zooplankton and conductivity on tropical Utricularia foliosa investment in carnivory. Aquatic Ecology 34, 137-142.

Guisande, C., Aranguren, N., Andrade-Sossa, C., Prat, N., Granado-Lorencio, C., Barrios, M. L., Bolivar, A., Nunez-Avellaneda, M., and Duque, S. R. (2004) Relative balance of the cost and benefit associated with carnivory in the tropical Utricularia foliosa. Aquatic Botany $80,271-282$.

Hodick, D. and Sievers, A. (1989) On the mechanism of trap closure of Venus flytrap (Dionaea muscipula Ellis). Planta 179, 32 - 42.

Humphrey W., Dalke A., and Schulten K. (1996) VMD: visual molecular dynamics. Journal of Molecular Graphics 14, 33 - 38.

Jobson, R. W. and Albert, V. A. (2002) Molecular rates parallel diversification contrasts between carnivorous plant sister lineages. Cladistics $18,127-136$.

Jobson, R. W., Nielsen, R., Laakkonen, L, Wikström, M., and Albert, V. A. (2004) Adaptive evolution of cytochrome $c$ oxidase: infrastructure for a carnivorous plant radiation. Proceedings of the National Academy of Sciences of the USA 101, 18064-18068.

Jobson, R. W., Playford, J., Cameron, K. M., and Albert, V. A. (2003) Molecular phylogenetics of Lentibulariaceae inferred from plastid rps16 intron and trnL-F DNA sequences: implications for character evolution and biogeography. Systematic Botany 28, 157-171.

Juniper, B. E., Robins, R. J., and Joel, D. M. (1989) The Carnivorous Plants. London, UK: Academic Press, Ltd.

Kadenbach, B (2003) Intrinsic and extrinsic uncoupling of oxidative phosphorylation. Biochimica et Biophysica Acta 1604, 77-94.

Knight, S. E. (1992) Costs of carnivory in the common bladderwort, Utricularia macrorhiza. Oecologia 89, 348 - 355.

Méndez, M. and Karlsson, P. S. (1999) Costs and benefits of carnivory in plants: insights from the photosynthetic performance of four carnivorous plants in a subarctic environment. Oikos 86, 105-112.

Mitchell, P. (1961) Coupling of phosphorylation to electron and hydrogen transfer by a chemi-osmotic type of mechanism. Nature $191,144-148$

Mitchell, P. (1966) Chemiosmotic coupling in oxidative and photosynthetic phosphorylation. Biological Reviews of the Cambridge Philosophical Society 41, 445-502.

Müller, K., Borsch, T., Legendre, L., Porembski, S., Theisen, I., and Barthlott, W. (2004) Evolution of carnivory in Lentibulariaceae and the Lamiales. Plant Biology 6, 447-490.

Ostermeier, C., Harrenga, A., Ermler, U., and Michel, H. (1997) Structure at $2.7 \AA$ resolution of the Paracoccus denitrificans two-subunit cytochrome $c$ oxidase complexed with an antibody FV fragment. Proceedings of the National Academy of Sciences of the USA 94, $10547-10553$.

Ribacka, C., Verkhovsky, M. I., Belevich, I., Bloch, D.A., Puustinen, A., and Wikström, M. (2005) An elementary reaction step of the proton pump is revealed by mutation of tryptophan-164 to phenylalanine in cytochrome $c$ oxidase from Paracoccus denitrificans. Biochemistry 44, 16502 - 16512.

Roberts, V.A. and Pique, M. E. (1999) Definition of the interaction domain for cytochrome $c$ on cytochrome $c$ oxidase. III. Prediction of the docked complex by a complete, systematic search. Journal of Biological Chemistry 274, 38051 - 38060

Richards, J. H. (2001) Bladder function in Utricularia purpurea (Lentibulariaceae): is carnivory important? American Journal of Botany $88,170-176$.

Saraste, M (1999) Oxidative phophorylation at the fin de siecle. Science $283,1488-1492$.
Schmidt, T. R., Wildman, D. E., Uddin, M., Opazo, J. C., Goodman, M., and Grossman, L. I. (2005) Rapid electrostatic evolution at the binding site for cytochrome $c$ on cytochrome $c$ oxidase in anthropoid primates. Proceedings of the National Academy of Sciences of the USA 102, 6379-6384.

Sharma, V., Puustinen, A., Wikström, M., and Laakkonen, L. (2006) Sequence analysis of the cbb3 oxidases and an atomic model for the Rhodobacter sphaeroides enzyme. Biochemistry 45, 57545765.

Skotheim, J. M. and Mahadevan, L. (2005) Physical limits and design principles for plant and fungal movements. Science 308, 1308 1310.

Soulimane, T., Buse, G., Bourenkov, G. P., Bartunik, H. D., Huber, R., and Than, M. E. (2000) Structure and mechanism of the aberrant ba(3)-cytochrome $c$ oxidase from Thermus thermophilus. EMBO Journal 17, 1766-1776

Stucki, J. (1980). The optimal efficiency and the economic degtrees of coupling of oxidative phosphorylation. European Journal of Biochemistry 109, 269-283.

Svensson-Ek, M., Abramson, J., Larsson, G., Tornroth, S., Brzezinski, P., and Iwata, S. (2002) The X-ray crystal structures of wild-type and EQ(I-286) mutant cytochrome $c$ oxidases from Rhodobacter sphaeroides. Journal of Molecular Biology 321, 329- 339.

Sydenham, P. H. and Findlay, G. P. (1973 a) Solute and water transport in the bladders of Utricularia. In Ion Transport in Plants (Anderson, W. P., ed.), New York: Academic Press, pp. 583 - 587.

Sydenham, P. H. and Findlay, G. P. (1973 b) The rapid movement of the bladders of Utricularia sp. Australian Journal of Biological Sciences 26, 1115 - 1126.

Sydenham, P. H. and Findlay, G. P. (1975) Transport of solutes and water by resetting bladders of Utricularia. Australian Journal of Plant Physiology 2, 335-351.

Tsukihara, T., Shimokata, K., Katayama, Y., Shimada, H., Muramoto, K., Aoyama, H., Mochizuki, M., Shinzawa-Itoh, K., Yamashita, E., Yao, M., Ishimura, Y., and Yoshikawa, S. (2003) The low-spin heme of cytochrome $c$ oxidase as the driving element of the proton-pumping process. Proceedings of the National Academy of Sciences of the USA 100, 15304-15309.

Wakefield, A. E., Gotelli, N. J., Wittman, S. E., and Ellison, A. M. (2005) Prey addition alters nutrient stoichiometry of the carnivorous plant Sarracenia purpurea. Ecology 86, 1737-1743.

Wikström, M. K. F. (1977) Proton pump coupled to cytochrome $c$ oxidase in mitochondria. Nature 266, 271 - 273.

\section{A. Albert}

Natural History Museum

University of Oslo

P.O. Box 1172 Blindern

0318 Oslo

Norway

E-mail: victor.albert@nhm.uio.no

Guest Editor: S. Porembski 